\title{
Sensibilidade corneana e secreção lacrimal após LASIK
}

\author{
Corneal sensivity and tear secretion after LASIK
}

\author{
Marcelo Guimarães Brandão Rêgo' ${ }^{1}$ \\ Adriano Jorge Mattoso Rodovalho' \\ André Adolfo Alves da Rocha ${ }^{2}$ \\ João J. Nassaralla Júnior ${ }^{3}$ \\ Belquiz R. Amaral Nassaralla ${ }^{4}$
}

\section{RESUMO}

Objetivo: Avaliar as alterações que ocorrem na sensibilidade corneana e secreção lacrimal após a cirurgia de "laser in situ keratomileusis" (LASIK). Métodos: Foram examinados 38 olhos de 19 pacientes, 9 homens e 10 mulheres submetidos à cirurgia de LASIK para correção de miopia com equivalente esférico médio de $-3,79 \mathrm{D}( \pm 1,29 \mathrm{D})$. A sensibilidade corneana foi medida com o estesiômetro de Cochet-Bonnet, na região central da córnea. A secreção lacrimal foi avaliada pelos testes de Schirmer I, secreção basal, medida do tempo de rotura do filme lacrimal, e sintomatologia apresentada. Os exames foram realizados antes e após 7, 30, 90, 180 e 270 dias da cirurgia ou até que os níveis pré-operatórios fossem atingidos. Resultados: Antes da cirurgia, o valor mediano da sensibilidade tátil corneana foi de $60,0 \mathrm{~mm}$ (variando de 50 a $60 \mathrm{~mm}$ ); o teste de Schirmer I apresentou mediana de 21,5 mm (variando de 10 a $30 \mathrm{~mm}$ ); o teste de secreção basal obteve mediana de $11,5 \mathrm{~mm}$ (variando de 6 a $20 \mathrm{~mm}$ ); o tempo de rotura do filme lacrimal alcançou mediana de 16,0 segundos (variando de 8 a 22 segundos). Todos os pacientes recuperaram seus valores préoperatórios de sensibilidade corneana e secreção lacrimal entre 90 e 180 dias após a cirurgia. Durante os 9 meses de seguimento, 5 pacientes (10 olhos) não apresentaram qualquer sintoma relacionado a olho seco. Conclusão: Após a cirurgia de LASIK a sensibilidade corneana e a secreção lacrimal podem ficar reduzidos por até 6 meses. Neste período, $73,6 \%$ dos pacientes apresentaram sintomas de olho seco. Estudos futuros são necessários para avaliar os efeitos destas alterações sobre a fisiologia corneana.

Descritores: Miopia/cirurgia; Ceratomileuse assistida por excimer laser in situ; Complicações pós-operatórias; Síndromes do olho seco/etiologia; Lagrimas/secreção; Córnea/ fisiologia

Estudo realizado no Instituto de Olhos de Goiânia, Goiânia, GO, Brasil.

${ }^{1}$ Médico residente do $3^{\circ}$ ano do Departamento de Oftalmologia do Hospital das Clínicas da Faculdade de Medicina de Ribeirão Preto - USP

${ }^{2}$ Ex-médico residente do Instituto de Olhos de Goiânia. ${ }^{3}$ Médico do Setor de Retina e Vítreo do Instituto de Olhos de Goiânia.

${ }^{4}$ Médica do Setor de Cirurgia Refrativa e Córnea do Instituto de Olhos de Goiânia, Doutora em Oftalmologia pela Universidade Federal de Minas Gerais.

Endereço para correspondência: Av. Caramuru, 2200/

134 - Ribeirão Preto (SP) CEP 14030-000

E-mail: marceloretina@globo.com

Recebido para publicação em 10.12.2001

Aceito para publicação em 11.09.2002 \begin{tabular}{l} 
INTRODUÇ̃̃o \\
\hline Nos últimos anos, a técnica de "Laser in situ keratomileusis" (LASIK) \\
tem se tornado bastante popular no tratamento de miopia, astigmatismo e \\
hipermetropia ${ }^{(1-3)}$. O LASIK associa a precisão do laser às vantagens da \\
cirurgia lamelar. Para corrigir miopia, pequena quantidade de tecido \\
corneano é removida da região central da córnea, resultando em seu \\
aplanamento. Quanto maior o erro refracional a ser corrigido, maior a pro- \\
fundidade da ablação e maior a quantidade de tecido corneano removido ${ }^{(1)}$. \\
Alguns estudos mostram que a profundidade da ablação(4-6) afeta a \\
regeneração dos nervos corneanos e a recuperação da sensibilidade. O \\
LASIK poupa o epitélio, a membrana de Bowman, o estroma superficial e \\
uma considerável porção do plexo nervoso anterior, já que os nervos \\
localizados no pedículo do disco não são afetados ${ }^{(7)}$. Os nervos corneanos \\
do restante da lamela são mecanicamente deslocados, mas sua estrutura
\end{tabular} 
mantém-se presente ${ }^{(8)}$. Terminada a ablação, o disco corneano é recolocado em sua posição original. Linna et $\mathrm{al}^{(7)}$ demonstraram que a porção distal do nervo seccionado degenera, deixando canais vazios através dos quais a porção proximal se regenera. Isto permite uma recuperação mais rápida e organizada do plexo nervoso.

Alguns estudos têm descrito hipoestesia corneana após o LASIK $^{(4-10)}$. Existem também autores ${ }^{(11)}$ que descreveram alterações na secreção lacrimal e sintomas de olho seco como efeito colateral desta técnica. No entanto, as informações que se tem sobre estas alterações e suas implicações clínicas são limitadas e controversas.

$\mathrm{O}$ ato de tocar a córnea provoca um dos mais importantes reflexos protetores do corpo humano: o piscar. O limiar de sensibilidade táctil corneana, especialmente no centro da córnea é bastante baixo, assim, alterações patológicas podem ser identificadas precocemente, auxiliando no diagnóstico, acompanhamento e mesmo prognóstico, de várias doenças corneanas. A perda ou redução da sensibilidade normal da córnea pode comprometer o reflexo do piscar, atrasar a cicatrização epitelial, reduzir o fluxo lacrimal, favorecer o aparecimento de ceratite neurotrófica, necrose corneana estéril e ceratite infecciosa ${ }^{(12-15)}$.

A diminuição da quantidade de secreção lacrimal observada em alguns trabalhos em pacientes operados pela técnica LASIK parece ser decorrente da lesão das terminações nervosas sensoriais da córnea ${ }^{(16)}$. Nas primeiras semanas após a cirurgia, observa-se instabilidade do filme lacrimal provavelmente como resultado do trauma ao epitélio corneano, toxicidade da medicação tópica, resposta inflamatória e diminuição da sensibilidade com menor freqüência do piscar ${ }^{(17)}$.

$\mathrm{O}$ estudo pretende avaliar as alterações que ocorrem na sensibilidade corneana e secreção lacrimal após a cirurgia de LASIK para a correção de miopia.

\section{MÉTODOS}

Em um estudo prospectivo, 38 olhos de 19 pacientes, 9 homens e 10 mulheres, com idade média de 32,7 anos, variando de 27 a 38 anos $( \pm 2,45)$, e equivalente esférico médio de $-3,79$ $\mathrm{D}( \pm 1,29)$, foram submetidos à técnica LASIK para a correção de miopia.

Para obtermos um padrão similar de tratamento (ablação corneana), pacientes com astigmatismo igual ou superior a 1,0 $\mathrm{D}$ foram excluídos do estudo. Também foram excluídos pacientes com refração instável, doenças oculares, anormalidades corneanas e palpebrais, história de diabetes, uso de medicação sistêmica e/ou tópica, doenças neurológicas, uso de lentes de contato por pelo menos um mês antes da cirurgia, cirurgia ocular prévia, sinais ou sintomas de olho seco e aqueles que faltaram a pelo menos uma das visitas pós-operatórios.

Os exames pré-operatórios incluíram: histórico médico ocular, medidas da acuidade visual sem e com correção, refração dinâmica e estática, biomicroscopia, paquimetria, tonometria, ceratometria, topografia corneana, microscopia especular da córnea, oftalmoscopia binocular indireta, estesiometria e exames para avaliar a secreção lacrimal. Os pacientes também foram questionados quanto a ter ou não sintomas de olho seco tais como: queimação, sensação de corpo estranho, ardência, prurido, fotofobia, hiperemia ocular, dor e dificuldade para movimentação das pálpebras. Todos foram informados sobre o método, natureza, objetivo e inocuidade dos exames a que seriam submetidos, bem como sobre a importância da assiduidade aos exames pós-operatórios.

A sensibilidade corneana foi medida na região central da córnea utilizando-se o estesiômetro de Cochet e Bonnet (LUNEAU, Paris, França). Este aparelho é composto de um corpo de metal com um monofilamento de nylon de $0,12 \mathrm{~mm}$ de diâmetro, e comprimento variável entre 0 e $60 \mathrm{~mm}$, que aplica uma pressão sobre a córnea entre 5 e $180 \mathrm{mg} / 0,0113 \mathrm{~mm}^{2}$. Para o exame, o estesiômetro era manuseado a prumo perpendicular à córnea, exercendo discreta pressão até obter um pequeno encurvamento (aproximadamente 5\%) do seu comprimento. Para evitar correntes de ar, o exame foi realizado em ambiente fechado com o paciente em decúbito dorsal, para que o fio de nylon do estesiômetro pudesse ser dirigido à córnea sem sofrer encurvamento, o que poderia falsear o resultado. $\mathrm{O}$ procedimento era repetido, com redução gradativa do comprimento do fio de 5 em $5 \mathrm{~mm}$. A cada toque, o paciente era questionado quanto a ter, ou não, sentido-o. O maior comprimento do fio capaz de estimular resposta ao estímulo era anotado em milímetros e considerado o limiar de sensibilidade da área central. Para confirmar a resposta do paciente, a área central foi testada três vezes, com intervalo mínimo de 60 segundos entre as medidas, e então tirada a média, que representou o limiar da sensibilidade. Conforme Cochet e Bonnet ${ }^{(14)}$, os valores considerados normais para a sensibilidade da córnea humana são aqueles superiores a $40 \mathrm{~mm}$ para a córnea central.

A secreção lacrimal foi avaliada através do teste de Schirmer I. O teste é feito sem a instilação de colírio anestésico. Utilizam-se tiras de papel de filtro (Whatman 41) de $5 \mathrm{~mm}$ de largura e $35 \mathrm{~mm}$ de comprimento, dos quais $5 \mathrm{~mm}$ são dobrados e inseridos no fundo de saco conjuntival, na união dos terços médio e externo da pálpebra inferior, com cuidado para não tocar a córnea. Durante o teste, solicitava-se ao paciente que olhasse para cima, sendo permitido o piscar. Após 5 minutos, a fita era retirada e o comprimento da área umedecida era medido, a partir da dobra. Esta prova mede a secreção basal e o reflexo trigeminal conjuntivo-lacrimal. Foram considerados normais, valores superiores a $15 \mathrm{~mm}$.

Como a colocação do papel de filtro no fundo de saco induz um lacrimejamento reflexo, utilizamos uma variante de teste de Schirmer I', com a instilação prévia de anestésico, na tentativa de medirmos apenas a secreção basal. Assim sendo, para avaliação da secreção lacrimal basal, 1 gota de cloridrato de proximetacaína $0,5 \%$ (Anestalcon ${ }^{\circledR}$ ) era instilada no fórnice inferior. A tira de papel de filtro (Whatman 41) era inserida no fundo de saco conjuntival, na união dos terços médio e externo da pálpebra inferior. Após 2 minutos a porção umedeci- 
da da fita era medida. São considerados normais valores superiores a $5 \mathrm{~mm}$.

O teste do tempo de rotura do filme lacrimal ("breakup time" - BUT) foi realizado através da instilação de 1 gota de fluoresceína sódica (Fluoresceína ${ }^{\circledR}$ - Allergan) no saco conjuntival inferior. Em seguida, solicitava-se ao paciente que piscasse várias vezes para distribuir a fluoresceína e procediase ao exame sob lâmpada de fenda. O filme lacrimal era avaliado através da luz com filtro azul de cobalto. O paciente mantinha a posição primária do olhar, sem piscar, e o intervalo entre o último piscar completo e o aparecimento de quebra do filme lacrimal era cronometrado. $\mathrm{O}$ exame era repetido por 3 vezes consecutivas e a média, anotada. Considerou-se como tempo de rotura normal, no mínimo 15 segundos.

Os procedimentos cirúrgicos foram realizados no Instituto de Olhos de Goiânia utilizando-se o excimer laser Technolas 217-C (Chiron Technolas/Bausch \& Lomb) e o microcerátomo ACS (Chiron Technolas/Bausch \& Lomb), no período de janeiro a dezembro de 1999. Em todos os casos, a zona óptica foi de $5 \mathrm{~mm}$ e placa de profundidade utilizada no microcerátomo foi de $160 \mu \mathrm{m}$. A técnica cirúrgica e o tratamento pós-operatório utilizados foram semelhantes aos descritos em estudos anteriores ${ }^{(2,4,18)}$.

A anestesia consistiu de duas gotas de cloridrato de proximetacaína tópica (Anestalcon ${ }^{\circledR} 0,5 \%$ ) 2 a 5 minutos antes da cirurgia. Todos os pacientes fizeram uso de 1 comprimido de sedativo oral (diazepam $5 \mathrm{mg}$ ) 30 minutos antes da cirurgia. Após assepsia da região periorbitária da face com solução de álcool iodado a $10 \%$, um campo cirúrgico de plástico é colocado e ajustado em volta do olho a ser operado cobrindo as margens palpebrais e os cílios. Um blefarostato é utilizado para obter máxima exposição do olho. Utilizando-se um marcador com três lâminas radiais assimétricas, a córnea é marcada com caneta de azul de metileno, facilitando o reposicionamento do disco lamelar ao término da cirurgia.

$\mathrm{O}$ anel de sucção é colocado sobre o olho, o pedal de suç̧ão acionado e a pressão intra-ocular medida com um tonômetro (Chiron Vision, Irvine, Califórnia, EUA) para garantir proporções ideais, em torno de $60 \mathrm{~mm} \mathrm{Hg}$. Um disco lamelar é confeccionado com o microcerátomo, na superfície anterior da córnea. O microcerátomo (Chiron Vision, Irvine, Califórnia, EUA) é encaixado nos trilhos do anel de sucção e ajustado na posição ideal. Pressionando-se o pedal, o microcerátomo desliza automaticamente em direção nasal confeccionando um disco lamelar corneano, pediculado. Com o disco lamelar pronto, mas ainda posicionado sobre o leito estromal, solicita-se ao paciente que fixe o olhar na luz vermelha de fixação coaxial do laser. O sistema de centralização é então ativado ligando-se a luz do laser e a luz do foco. Com o paciente fixando na luz vermelha, tem-se a posição exata do local a ser tratado. O sistema "eye tracker" é então ativado e o paciente solicitado a permanecer fixando a luz. Assim, o disco lamelar é levantado com uma espátula, repousado sobre a conjuntiva medial, seu pedículo protegido por uma esponja cirúrgica e o pedal do laser acionado. A ablação com o excimer laser é realizada no leito estromal, e a centralização mantida pela fixação e garantida pelo sistema "eye tracker". Segue-se uma ablação refrativa na região central do leito estromal exposto, cuja quantidade de tecido removido depende do erro refracional a ser corrigido, o que é calculado pelo aparelho de excimer laser.

Terminada a ablação, o disco corneano é então reposicionado e o leito estromal cuidadosamente irrigado com solução salina balanceada para remover impurezas ou possíveis células epiteliais implantadas na interface. Com uma esponja cirúrgica umedecida, o disco é realinhado, acompanhando a distribuição das marcas radiais de azul de metileno, sem necessidade de sutura. Para facilitar a aderência do disco lamelar ao leito estromal aguarda-se 3 minutos. Em seguida, para certificar-se de que a adesão está suficiente, pressiona-se a periferia da córnea com uma espátula e observava-se se as estrias formadas se propagam no disco corneano (teste da estria). Uma gota de colírio tobramicina/ dexametasona $\left(\right.$ Tobradex $^{\circledR}$ ) e outra de cetorolac de trometamina $0,5 \%\left(\right.$ Acular $\left.^{\circledR}\right)$ são instiladas no fundo de saco. Em seguida, o mesmo procedimento é repetido no olho contralateral.

Ao final da cirurgia, protetores transparentes fenestrados eram colocados sobre os olhos e presos à pele com fita cirúrgica. Nenhum paciente teve o olho ocluído. Os pacientes eram solicitados a iniciar a terapêutica pós-operatória com Tobradex ${ }^{\circledR}$ colírio 4 vezes ao dia durante 8 dias.

Os exames para a avaliação da sensibilidade táctil corneana e da secreção lacrimal foram realizados antes e após 7, 30, 90,180 e 270 dias da cirurgia ou até que os níveis pré-operatórios fossem atingidos.

O teste não-paramétrico de Friedman e o teste de comparações múltiplas foram utilizados na análise estatística dos dados de sensibilidade corneana, Schirmer I, Schirmer I' e BUT (variáveis de resposta), considerando cada paciente como um bloco de observação, caracterizando um ensaio clínico com medidas repetidas. O teste estatístico foi escolhido devido ao tamanho da amostra e porque a informação observada é do tipo ordinal - escores. Foram considerados os escores nos instantes pré e pós-operatórios (7, 30, 90, 180 e 270 dias $)$. As medianas em cada instante foram adotadas como estimativas. O nível de significância $(\alpha)$ adotado foi de $5 \%$.

Cada paciente assinou um termo de consentimento livre e esclarecido, segundo protocolo aprovado pela Comissão de Ética do Instituto de Olhos de Goiânia.

\section{RESULTADOS}

O quadro 1 apresenta as medianas observadas nos pacientes no pré- e nos instantes pós-operatórios observados em cada variável de resposta estudada. O teste de Friedman revelou a existência de diferenças estatísticas entre os escores observados, ao longo do tempo, nas variáveis de reposta.

Para a sensibilidade corneana observou-se que no préoperatório o valor mediano foi de $60,0 \mathrm{~mm}$ que, no pós-operatório, apresentou escores zero após 7 dias, 10,0 $\mathrm{mm}$ após 30 


\begin{tabular}{|c|c|c|c|c|c|c|}
\hline \multirow[t]{2}{*}{ Variável de resposta } & \multirow[t]{2}{*}{ Pré-operatório } & \multicolumn{5}{|c|}{ Pós-operatório } \\
\hline & & 7 dias & 30 dias & 90 dias & 180 dias & 270 dias \\
\hline Sensibilidade & $60,0^{b}$ & $0,0^{a}$ & $10,0^{a}$ & $32,5^{b}$ & $60,0^{b}$ & $60,0^{b}$ \\
\hline Schirmer I & $21,5^{d}$ & $5,0^{a}$ & $8,0^{b}$ & $15,0^{c}$ & $21,0^{d}$ & $21,5^{d}$ \\
\hline Schirmer I' & $11,5^{c}$ & $3,0^{a}$ & $5,5^{b}$ & $9,0^{c}$ & $11,5^{c}$ & $11,5^{c}$ \\
\hline BUT $^{*}$ & $16,0^{c}$ & $4,0^{a}$ & $8,5^{b}$ & $10,5^{c}$ & $15,5^{c}$ & $16,0^{c}$ \\
\hline
\end{tabular}

dias, 32,5 mm após 90 dias e $60,0 \mathrm{~mm}$ após 180 e 270 dias. A análise estatística mostrou que após 90 dias não havia diferença estatisticamente significante com o pré-operatório, considerando-se que a sensibilidade corneana tenha retornado ao estado pré-operatório, clinicamente, após 180 dias.

A mediana da medida de Schirmer I no pré-operatório foi de $21,5 \mathrm{~mm}$. No pós-operatório, os valores medianos observados para os escores foram de $5,0 \mathrm{~mm}$ após o $7^{\circ}$ dia, $8,0 \mathrm{~mm}$ no $30^{\circ}$ dia, $15,0 \mathrm{~mm}$ no $90^{\circ} \mathrm{dia}, 21,0 \mathrm{~mm}$ no $180^{\circ}$ dia e $21,5 \mathrm{~mm}$ após 270 dias. A análise estatística mostrou que no $90^{\circ}$ dia a medida de Schirmer I ainda apresentava redução significante em relação ao pré-operatório, com a recuperação clínica dos valores ocorrendo após 180 dias.

A medida de Schirmer I' apresentou um comportamento diferente da medida original, e a análise estatística mostrou que no $90^{\circ}$ dia a redução apresentada da secreção lacrimal não era estatisticamente significante comparado ao pré-operatório; clinicamente, a recuperação dos valores deu-se após 180 dias. A medida de Schirmer I' apresentou escore $11,5 \mathrm{~mm}$ no pré-operatório, 3, $0 \mathrm{~mm}$ após 7 dias, 5,5 $\mathrm{mm}$ após 30 dias, $9,0 \mathrm{~mm}$ após 90 dias e 11,5 $\mathrm{mm}$ após 180 e 270 dias.

O valor mediano de BUT foi de 16,0 segundos no préoperatório, 4,0 segundos após 7 dias, 8,5 após 30 dias, 10,5 após 90 dias, 15,5 após 180 dias e 16,0 após 270 dias. No $90^{\circ}$ dia o teste estatístico mostrou haver concordância com os resultados obtidos pela medida de Schirmer I, ou seja, após 90 dias, os níveis de BUT estavam estatisticamente restabelecidos; clinicamente, isso ocorreu após 180 dias.

O quadro 1, além de apresentar as estimativas de efeito, mostra, também, que houve diferença estatística entre os valores pré-operatórios e o pós-operatório de 7 e 30 dias, evidenciando a queda de sensibilidade e de secreção lacrimal, para todas as variáveis de resposta estudadas. Somente o teste de Schirmer I apresentou diferença estatística também no $90^{\circ}$ dia. O gráfico 1 ilustra estes resultados.

\section{DISCUSSÃO}

Nos dias de hoje, tornou-se prática comum a realização de cirurgia refrativa para a correção de miopia, astigmatismo ou hipermetropia, principalmente através da técnica de "laser in situ keratomileusis".

O presente estudo confirma que alterações na secreção lacrimal, sensibilidade corneana e sintomas de olho seco, são comuns após a cirurgia de LASIK. Nesta técnica, um disco corneano ligado à córnea por um pedículo, é obtido com auxílio do microcerátomo. Depois da ceratotomia superficial realizada pelo microcerátomo, obtém-se um disco corneano composto por epitélio, membrana de Bowman e estroma anterior. Durante a ceratotomia, os nervos estromáticos superficiais são cortados e as terminações nervosas do leito corneano são expostas à fotoablação pelo excimer laser. O resultado mostra que houve redução estatisticamente significante tanto da secreção lacrimal quanto da sensibilidade táctil corneana na avaliação pós-operatória de 1 semana e 30 dias.

Aos 6 meses de pós-operatório, observou-se restabelecimento dos valores obtidos nos testes de avaliação táctil corneana e secreção lacrimal, o que sugere a recuperação clínica de todos os parâmetros avaliados após esse período. O teste de Schirmer I mostrou retorno mais lento aos valores prévios, o que indica que a secreção reflexa apresentou recuperação mais lenta que a basal. Após 3 meses, todos os parâmetros permaneciam reduzidos em relação ao pré-operatório, mas sem significância estatística com exceção do teste de Schirmer I.

Verificou-se ainda que apesar de todos os pacientes terem apresentado alterações na sensibilidade e secreção lacrimal, 5 deles $(26,3 \%)$ não referiram qualquer sintoma quando questionados sobre olho seco durante todo o período de avaliação pós-operatória.

Benitez-del-Castillo et al. ${ }^{(11)}$, analisando 48 olhos após o LASIK, relataram que a sensibilidade corneana e o filme lacrimal retornaram aos seus valores pré-operatórios somente após 9 meses da cirurgia. Porém, seus pacientes apresentavam

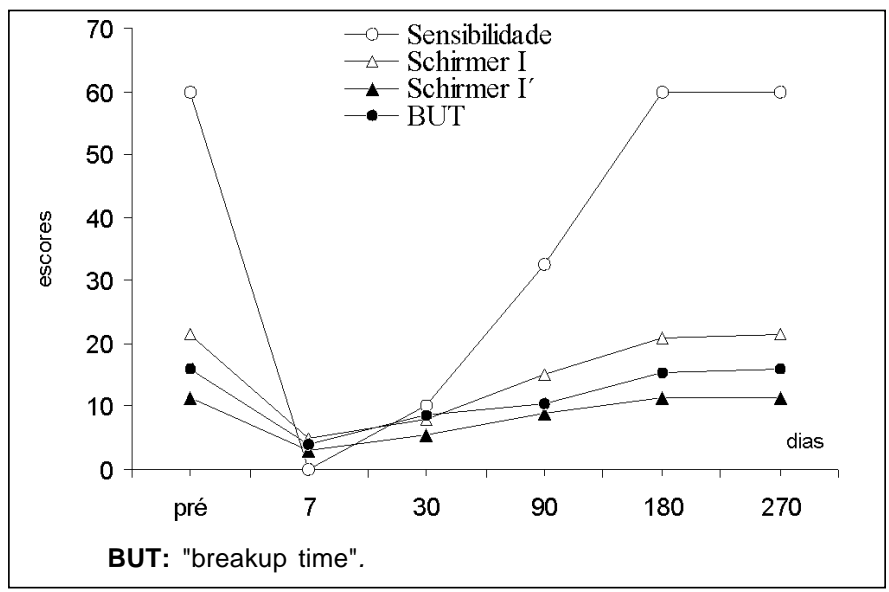

Gráfico 1 - Medianas dos escores observados no pré-e pós-operatórios, do estudo de sensibilidade corneana e secreção lacrimal 
elevada miopia (até -13,00D) o que necessita de uma ablação corneana mais profunda e consequentemente, maior quantidade de tecido corneano é lesado. Além disso, em seu estudo, não foram excluídos os pacientes usuários de lente de contato, o que pode ter influenciado o resultado.

Em estudo anterior ${ }^{(4)}$, demonstramos variação significativa na recuperação da sensibilidade corneana em pacientes submetidos ao LASIK para correção de diferentes erros refracionais, isto é, a recuperação variou de acordo com a profundidade da ablação. Após 180 dias, houve recuperação total da sensibilidade tátil corneana nos pacientes com erro refracional abaixo de -8,00D, e após 270 dias naqueles com erro refracional acima de -8,00D. Kim e $\mathrm{Kim}^{(5)}$ relacionaram profundidade de ablação com atraso na recuperação da sensibilidade. No entanto, incluíram pacientes tratados não apenas para miopia, mas também para astigmatismo miópico composto, o que implica diferentes padrões de ablação, aumentando o número de variáveis a serem analisadas. Eles observaram que, nos casos de altos erros refracionais (ablações superiores a $100 \mu \mathrm{m}$ ), a sensibilidade corneana não recuperou seus níveis pré-operatórios, mesmo após 6 meses da cirurgia. Infelizmente, o seguimento por eles realizado foi de apenas 180 dias e não até a completa recuperação.

Yu et al. ${ }^{(17)} \mathrm{em}$ seu estudo com 96 olhos mostraram redução dos valores de secreção lacrimal 1 semana e 1 mês após o LASIK. O teste de Schirmer reduziu de 13,32 $\pm 10,67$ para 11,18 $\pm 9,81$, e o teste Schirmer I, de 8,04 $\pm 8,48$ para 5,67 $\pm 6,87$ após 1 semana. Trinta dias após a cirurgia, o teste de Schirmer diminuiu para 10,83 $\pm 10,02$ e o Schirmer I, para 5,62 $\pm 6,24$. Esse difere quanto tempo de seguimento pós-operatório, sendo avaliado até 1 mês de LASIK e também incluindo pacientes com sintomas de olho seco.

A desenervação corneana tem sido relacionada a sintomas de olho seco após LASIK. Os resultados mostram que realmente existe grande risco para tais sinais e sintomas após esta cirurgia, sendo maior nos pacientes com maiores erros refracionais, isto é, com ablações mais profundas.

\section{CONCLUSÃO}

As alterações verificadas no filme lacrimal e sensibilidade tátil corneana, principalmente no primeiro mês após o LASIK, sugerem um seguimento pós-operatório cuidadoso no sentido de observar e tratar possíveis sinais de olho seco. Estudos futuros são necessários para avaliar os efeitos dessas alterações na fisiologia da córnea e suas implicações clínicas em longo prazo.

\section{AGRADECIMENTOS}

Ao Professor Doutor Antônio Luiz Rodrigues Júnior, do Departamento de Medicina Social da Faculdade de Medicina de Ribeirão Preto - USP, pelo valioso auxílio na análise estatística dos dados deste trabalho.

\begin{abstract}
Purpose: To analyze the changes in corneal sensitivity and tear secretion after "laser in situ keratomileusis" (LASIK). Methods: Thirty-eight eyes from nineteen patients, 9 men and 10 women, were submitted to myopic LASIK. The mean spherical equivalent before surgery was $-3.79 \mathrm{D}( \pm 1.29)$. Corneal sensitivity was measured with a Cochet-Bonnet esthesiometer in the central region of the cornea. Tear secretion was evaluated by the Schirmer test, basal secretion, breakup time test and symptoms. Examinations were performed before and 7, 30, 90, 180, and 270 days after surgery, or until full recovery. Results: Median preoperative corneal sensitivity was $60 \mathrm{~mm}$ (range, 50 to $60 \mathrm{~mm}$ ); median Schirmer test was $21.5 \mathrm{~mm}$ (range, 10 to $30 \mathrm{~mm}$ ); median basal secretion was $11.5 \mathrm{~mm}$ (range, 6 to $20 \mathrm{~mm}$ ); median breakup time test was 16.0 seconds (range, 8 to 22 seconds). All patients recovered their preoperative corneal sensitivity and tear secretion values between 90 to 180 days after surgery. During 9 months of follow-up, 5 patients (10 eyes) did not present any dry eye symptom. Conclusion: After LASIK, the corneal sensitivity and tear secretion are decreased as long as for 6 months. During the postoperative period, $73.6 \%$ of the patients had dry eye symptoms. Further studies are required to best evaluate the effects on the corneal physiology.
\end{abstract}

Keywords: Myopia/surgery; Laser in situ keratomileusis; Postoperative complications; Dry eye syndromes/etiology; Tears/secretion; Cornea/physiology

\section{REFERÊNCIAS}

1. Pallikaris IG, Papatzanaki ME, Siganos DS, Tsilimbaris MK. A corneal flap technique for laser in situ keratomielusis. Human studies. Arch Ophthalmol 1991;109:1699-702.

2. Nassaralla BA, Nassaralla JJ. Laser in situ keratomileusis after penetrating keratoplasty. J Refract Surg 2000;16:431-7.

3. Ibrahim O. Laser in situ keratomileusis for hyperopia and hyperopic astigmatism. J Refract Surg 1998;14(2 Suppl):S179-82.

4. Nassaralla BA, Nassaralla Jr. JJ. Human corneal sensitivity after LASIK [abstract]. Invest Ophthalmol Vis Sci 2001;42:3224.

5. Kim WS, Kim JS. Change in corneal sensitivity following laser in situ keratomileusis. J Cataract Refract Surg 1999;25:368-73.

6. Ishikawa TI, Park SB, Cox C, Del Cero M, Aquavella JV. Corneal sensation following excimer laser photorefractive keratectomy in humans. J Refract Corneal Surg 1994;10:417-22.

7. Linna TU, Pérez-Santonja JJ, Tervo KM, Sakla HF, Alio-Sanz JL, Tervo TMT. Recovery of corneal nerve morphology following laser in situ keratomileusis. Exp Eye Res 1998;66:755-63.

8. Kanellopoulous AJ, Pallikaris IG, Donnenfeld ED, Detorakis S, Koufala K, Perry HD. Comparison of corneal sensation following photorefractive keratectomy and laser in situ keratomileusis. J Cataract Refract Surg 1997;23:34-8.

9. Pérez-Santonja JJ, Sakla HF, Cardona D, Chipont E, Alió JL. Corneal sensitivity after photorefractive keratectomy and laser in situ keratomileusis for low myopia. Am J Ophthalmol 1999;127:497-504.

10. Linna TU, Vesaluoma MH, Pérez-Santonja JJ, Petroll WM, Alio JL, Tervo TM. Effect of myopic LASIK on corneal sensitivity and morphology of subbasal nerves. Invest Ophthalmol Vis Sci 2000;41:393-7.

11. Benitez-del-Castillo JM, Del Rio T, Iradier T, Hernández JL, Castillo A, Garcia-Sanchez J. Decrease in tear secretion and corneal sensitivity after laser in situ. Cornea 2001;20:30-2.

12. Martin XD, Safran AB. Corneal hypoesthesia. Surv Ophthalmol 1988;33:28-40. 
13. Beuerman RW, Schimmelpfennig B. Sensory denervation of the rabbit cornea affects epithelial properties. Exp Neurol 1980;69:196-201.

14. Cochet P, Bonnet R. L'ésthésie cornéenne. Sa mesure clinique. Ses variations physiologiques et pathologiques. Clin Ophtalmol 1960;4:3-27.

15. Schimmelpfennig B. Nerve structures in human central corneal epithelium. Graefes Arch Clin Exp Ophthalmol 1982;218:14-20.
16. Tervo K, Latvala TM, Tervo TMT. Recovery of corneal inervationn following photorefrective keratoablation. Arch Ophthalmol 1994;112:1466-70.

17. Yu EY, Leung A, Rao S, Lam DS. Effect of laser in situ keratomileusis on tear stability. Ophthalmology 2000;107:2131-5.

18. Barbosa RL, Nassaralla BA. LASIK para anisometropia associada à ambliopia em pacientes adultos. Rev Bras Oftalmol 2001;60:97-103.

\section{(d) (J) XxxII Congresso Brasileiro de Oftalmologia}

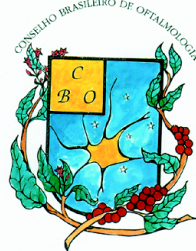

\section{0 a 13 de Setembro de 2003 - Salvador - BA CENTRO DE CONVENÇÕES DABAHIA}

\section{CONVIDADOS ESTRANGEIROS CONFIRMADOS}

$\begin{array}{ll}\text { Enique Graue - México } & \text { Miguel Burnier - Canadá } \\ \text { Narsing Rao - EUA } & \text { Ronald R. Krueger - EUA } \\ \text { Mark J. Mannis - EUA } & \text { Todd P. Margolis - EUA } \\ \text { Mark S. Blumenkranz - EUA } & \end{array}$

CONFERÊNCIA CBO - "RETINA NO SÉCULO XXI" - Dr. Marcos Avila

TAXA DE INSCRIÇÃO

\begin{tabular}{|lccc|}
\hline Categoria & $\begin{array}{c}\text { de } 16 / 04 \\
\text { até } 16 / 06\end{array}$ & $\begin{array}{c}\text { de 17/06 } \\
\text { até } 31 / 07\end{array}$ & $\begin{array}{c}\text { no } \\
\text { local }\end{array}$ \\
Sócios CBO & $\mathrm{R} \$ 290,00$ & $\mathrm{R} \$ 350,00$ & $\mathrm{R} \$ 460,00$ \\
Não Sócios & $\mathrm{R} \$ 560,00$ & $\mathrm{R} \$ 610,00$ & $\mathrm{R} \$ 720,00$ \\
Residentes / Acadêmicos & $\mathrm{R} \$ 145,00$ & $\mathrm{R} \$ 175,00$ & $\mathrm{R} \$ 230,00$ \\
Outros Profissionais da Área de Saúde & $\mathrm{R} \$ 145,00$ & $\mathrm{R} \$ 175,00$ & $\mathrm{R} \$ 230,00$ \\
Auxiliares em Oftalmologia & $\mathrm{R} \$ 75,00$ & $\mathrm{R} \$ 95,00$ & $\mathrm{R} \$ 110,00$ \\
Acompanhantes & $\mathrm{R} \$ 75,00$ & $\mathrm{R} \$ 95,00$ & $\mathrm{R} \$ 110,00$ \\
\hline
\end{tabular}

\section{ATENÇÃO}

DATA - LIMITE PARA ENVIO DE TRABALHOS CIENTÍFICOS: 15/04/03 - VÍDEOS: 17/06/03

\section{INFORMAÇ̃̃ES:}

SECRETARIA EXECUTIVA / ORGANIZAÇÃO:

INTERLINK - Consultoria \& Eventos

Tel.: (71) 336-5644 • Fax: (71) 336-5633

E-mail: oftalmo@interlinkeventos.com.br

Home Page: www.interlinkeventos.com.br

\section{AGÊNCIA DE TURISMO OFICIAL - INTERLINK TURISMO}

Tel.: (71) 336-5644 • Fax: (71) 336-5855

E-mail: it @interlinkeventos.com.br

Home Page: www.interlinkeventos.com.br

CBO: (1 1) 3266-4000 • Fax: (1 1) 3171-0953 • E-mail: eventos@cbo.com.br 